\title{
Reliability analysis of circular concrete-filled steel tube with material and geometrical nonlinearity
}

\author{
Tengfei XU, Tianyu XIANG*, Yulin ZHAN, Renda ZHAO \\ School of Civil Engineering, Southwest Jiaotong University, Chengdu 610031, China
}

\begin{abstract}
Most of the previous research on concrete-filled steel tube is restricted to a deterministic approach. To gain clear insight into the random properties of circular concrete-filled steel tube, reliability analysis is carried out in the present study. To obtain the structural nonlinear response and ultimate resistance capacity, material and geometrical nonlinear analysis of circular concrete-filled steel tube is performed with a three-dimensional degenerated beam element. Then we investigate the reliability of concrete-filled steel tube using the first-order reliability method combined with nonlinear finite element analysis. The influences of such parameters as material strength, slenderness, initial geometrical imperfection, etc. on the reliability of circular concrete-filled steel tube column are studied. It can be concluded that inevitable random fluctuation of those parameters has significant influence on structural reliability, and that stochastic or reliability methods can provide a more rational and subjective evaluation on the safety of CFT structures than a deterministic approach.
\end{abstract}

Key words: concrete-filled steel tube; reliability; material nonlinearity; geometrical nonlinearity

(C) 2012 JMT. All rights reserved.

\section{Introduction}

$\mathrm{C}$ oncrete-filled steel tube (CFT), which combines the advantages of steel and concrete, has been developed as an excellent structural element for building columns, bridge piers, and arch ribs throughout worldwide engineering practices. Since the steel tube can serve as a form for casting of core concrete, CFT structures possess economical merits in construction. Moreover, compared with hollow steel tube, core concrete can prevent buckling of steel tube, which may improve the compressive stability enormously. The most attractive advantage of concrete-filled steel tube structures is that the element exhibits an excellent compressive resistance capacity, ductility, and energy dissipation ability owing to the confining effect provided by steel tube.

A systematical and comprehensive collection of the research work about CFT structures can be found in Ref. [1]. In the previous research, the deterministic method plays a key role in the establishment of the design theory of CFT structures. However, it should be noted that those parameters, which influence the me-

Received Mar. 16, 2012; revision accepted Apr. 17, 2012

*Corresponding author. E-mail: tyxiang@home.swjtu.edu.cn (T.Y. XIANG)

(C) 2012 JMT. All rights reserved

doi: 10.3969/j.issn.2095-087X.2012.03.003 chanical behaviors of CFT structures, always possess a random nature. Therefore, it may be logical to involve those uncertainties in the analysis of CFT structures. In the present study, the authors intend to investigate the reliability of CFT structures with the combination of the first-order reliability method (FORM) and nonlinear finite element analysis.

Previous studies [2-4] have indicated that FORM is sufficiently accurate for engineering application. With linearization of the failure surface at the design point, which is the point closest to the origin in the standard normal space, FORM gives the first-order estimate of the probability of failure, $P_{f}=\Phi(-\beta)$, where $\beta$, the reliability index, is the distance between the origin and design points in the standard normal space, and $\Phi$ is the cumulative distribution function of standard normal distribution.

The reliability of nonlinear structures has received an increasing interest in the past twenty years, and a great amount of important research has been continuously contributed to this subject. The reliability of geometrical nonlinear structures has been studied in Refs. [5-6], in which FORM and SORM (second-order reliability method) are both used to calculate the reliability index, and the response gradient of the limit state function to random variables is directly derived from the equilibrium equation. Impollonia and Sofi [7] studied the reliability of geometrical nonlinear structures by adopting the response surface method (RSM). For reliability analysis of concrete structures, to avoid the underdesign 
or overdesign of RC structures, material nonlinearity should not be neglected [8]. Teigen et al. [9] presented a method based on the perturbation technique for reliability analysis of reinforced concrete planar frame. Biondini et al. [10] studied the reliability of material and geometrical nonlinear reinforced concrete and prestressed concrete structures by Monte Carlo method, and analyzed the reliability of an existing prestressed concrete arch bridge. Through combining fiber element analysis and Monte Carlo method, Lee and Mosalam [11] also studied the reliability of RC structures. With FORM and RSM, Val et al. [12] studied the reliability of nonlinear reinforced concrete structures. Soares et al. [13] also investigated the reliability of nonlinear reinforced concrete structures by FORM and RSM. In Refs. [12-13], the ultimate capacity of reinforced concrete structure with material and geometrical nonlinearity are mainly concerned. To determine the ultimate limit state, Val et al. [12] assumed that if the nonlinear iteration did not converge, the ultimate limit state was reached. A similar way was adopted by Soares et al. [13], and the singularity of the tangent stiffness matrix was used to characterize the ultimate limit state.

In the present study, a nonlinear analysis program for concrete structures with degenerated beam elements developed by Xiang et al. [14] is adopted to investigate the nonlinear behaviors of CFT structure. For confined concrete, the stress-strain relationship proposed by $\mathrm{Xu}$ et al. [15] is used. To investigate the influence of geometrical nonlinearity, updated Lagrangian formulation will be employed. When strong material nonlinearity is involved in finite element analysis, the failure surface may no longer be smooth, which may lead to the convergence difficulty in the FORM algorithm. To improve the convergence dilemma, the reliability scheme is built by coupling nonlinear finite element analysis with RSM in the present study.

\section{Nonlinear finite element analysis of cir- cular concrete-filled steel tube}

\subsection{Formulation of degenerated beam element}

The position of any point in the element shown in Fig. 1 can be given by

$$
\boldsymbol{X}=\sum_{i=1}^{2} N_{i}(r)\left(\boldsymbol{X}_{i}+y^{\prime} \boldsymbol{V}_{2}+z^{\prime} \boldsymbol{V}_{3}\right)
$$

in which $\boldsymbol{X}$ is the coordinates of any point in the element; $\boldsymbol{X}_{i}$ is the coordinates of node $i(i=1,2) ; \boldsymbol{V}_{1}, \boldsymbol{V}_{2}$ and $\boldsymbol{V}_{3}$ form the local orthogonal coordinate system illustrated in Fig. 1; $y^{\prime}$ and $z^{\prime}$ are the local coordinates in $\boldsymbol{V}_{2}$ and $\boldsymbol{V}_{3}$ directions, respectively; the interpolation function $N_{i}(r)$ is

$$
N_{i}(r)=\frac{1}{2}\left(1+r_{i} r\right)
$$

where $r_{i}$ is the isoparametric coordinate of node $i$.

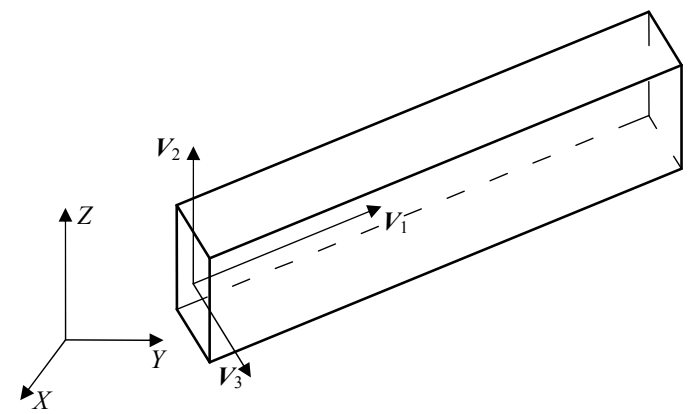

Fig. 1 The beam element

The local orthogonal coordinate system can be constructed in the following way

$$
\boldsymbol{V}_{3}=\boldsymbol{V}_{1} \times \boldsymbol{V}_{2},
$$

in which $V_{1}$ is expressed as

$$
\boldsymbol{V}_{1}=\left(\left(x_{2}-x_{1}\right) / L\left(y_{2}-y_{1}\right) / L\left(z_{2}-z_{1}\right) / L\right)^{\mathrm{T}} \text {, }
$$

where $L$ is the length of the element. With the given $\boldsymbol{V}_{2}$, $V_{3}$ can be finally determined through Eq. (3).

The displacement field can be expressed as

$$
\boldsymbol{U}=\sum_{i=1}^{2} N_{i}(r)\left[\boldsymbol{U}_{i}+\boldsymbol{U}_{\alpha}\left(y^{\prime}, z^{\prime}\right)\right]
$$

where $\boldsymbol{U}$ is the displacement vector at any point in the element, $\boldsymbol{U}_{i}$ is the nodal displacement vector at node $i$, and $\boldsymbol{U}_{\alpha}\left(y^{\prime}, z^{\prime}\right)$ is the relative nodal displacement produced by normal rotations. With a hypothesis of the plane section, $\boldsymbol{U}_{\alpha}\left(y^{\prime}, z^{\prime}\right)$ can be expressed as

$$
\left(\begin{array}{c}
u_{i}(\alpha) \\
v_{i}(\alpha) \\
w_{i}(\alpha)
\end{array}\right)=\left|\begin{array}{ccc}
\boldsymbol{V}_{1} & \boldsymbol{V}_{2} & \boldsymbol{V}_{3} \\
\alpha_{i 1}^{\prime} & \alpha_{i 2}^{\prime} & \alpha_{i 3}^{\prime} \\
0 & y^{\prime} & z^{\prime}
\end{array}\right|,
$$

where $\alpha_{i 1}^{\prime}, \alpha_{i 2}^{\prime}$, and $\alpha_{i 3}^{\prime}$ are the normal rotations about local axes of $\boldsymbol{V}_{1}, \boldsymbol{V}_{2}$, and $\boldsymbol{V}_{3}$.

With displacement transformation relation, Eq. (6) can be written in terms of the normal rotations about global axes as 


$$
\begin{aligned}
& \left(\begin{array}{c}
u_{i}(\alpha) \\
v_{i}(\alpha) \\
w_{i}(\alpha)
\end{array}\right)= \\
& {\left[\begin{array}{ccc}
0 & y^{\prime} n_{2}+z^{\prime} n_{3} & -y^{\prime} m_{2}-z^{\prime} m_{3} \\
-y^{\prime} n_{2}-z^{\prime} n_{3} & 0 & y^{\prime} l_{2}+z^{\prime} l_{3} \\
y^{\prime} m_{2}+z^{\prime} m_{3} & -y^{\prime} l_{2}-z^{\prime} l_{3} & 0
\end{array}\right]\left(\begin{array}{c}
\alpha_{i 1} \\
\alpha_{i 2} \\
\alpha_{i 3}
\end{array}\right),}
\end{aligned}
$$

in which

$$
\left(\begin{array}{lll}
l_{2} & m_{2} & n_{2}
\end{array}\right)^{\mathrm{T}} \text { and }\left(\begin{array}{lll}
l_{3} & m_{3} & n_{3}
\end{array}\right)^{\mathrm{T}}
$$

represent the direction cosine of $\boldsymbol{V}_{2}$ and $\boldsymbol{V}_{3}$ in the global coordinate system, respectively. Then, Eq. (5) can be rewritten as

$$
\boldsymbol{U}=\sum_{i=1}^{2} N_{i}(r)\left(\boldsymbol{U}_{i}+\boldsymbol{\Phi} \boldsymbol{a}_{i}\right)
$$

where

$$
\boldsymbol{\Phi}=\left[\begin{array}{ccc}
0 & y^{\prime} n_{2}+z^{\prime} n_{3} & -y^{\prime} m_{2}-z^{\prime} m_{3} \\
-y^{\prime} n_{2}-z^{\prime} n_{3} & 0 & y^{\prime} l_{2}+z^{\prime} l_{3} \\
y^{\prime} m_{2}+z^{\prime} m_{3} & -y^{\prime} l_{2}-z^{\prime} l_{3} & 0
\end{array}\right],
$$

and $\boldsymbol{\alpha}_{i}$ is the normal rotation vector about the global axes.

\subsection{Constitutive model of concrete and steel}

In the degenerated beam element, the cross-section will be subdivided into a series of pieces, and the axial stress of every piece will be computed with the onedimensional constitutive model [14]. In this section, the uniaxial constitutive model of confined concrete and steel will be discussed.

\subsubsection{Confined concrete}

The experimental studies have revealed that the confining effect of steel tube can increase the compressive strength and ductility of concrete. To describe a complete curve of one-dimensional stress-strain relation of concrete confined by steel tube, several characteristic points must be identified. For the ascending branch, the compressive strength of confined concrete $f_{c c}$, the corresponding strain $\varepsilon_{c c}$, and the initial elastic modulus of concrete $E_{0}$ should be determined. The compressive strength of confined concrete can be written as

$$
f_{c c}=f_{c}+\alpha m f_{r p},
$$

where $f_{c}$ is the compressive strength of unconfined concrete, $f_{r p}$ is the average radial pressure acted on the core concrete, and $m$ is an empirical constant which is assumed to be 4.2 in the present study. It has been found that the average radial pressure $f_{r p}$ depends on the widthto-thickness ratio $(D / t)[16]$. An empirical formula of $f_{r p}$ presented by $\mathrm{Xu}$ et al. [15] is adopted

$$
\frac{f_{r p}}{f_{y}}=0.3111\left(\frac{D}{t}-2\right)^{-1.027} \text {. }
$$

Since the confining stress of steel tube to core concrete will decrease gradually with increasing load eccentricity [16-17], a reduction parameter $\alpha$ is introduced into Eq. (10), which is given as [15]

$$
\alpha=\left\{\begin{array}{lc}
\left(1-\frac{2 e}{D}\right) \exp \left(-\frac{2 e}{D}\right) & \text { for } \frac{2 e}{D}<1, \\
0 & \text { for } \frac{2 e}{D} \geq 1,
\end{array}\right.
$$

where $e$ represents the load eccentricity, and can be expressed as $M / N$ ( $M$ and $N$ are the moment and axial force acted on the given section, respectively).

$\varepsilon_{c c}$, the strain of the confined concrete corresponding to maximum stress $f_{c c}$, is defined as

$$
\varepsilon_{c c}=\frac{f_{c c}}{f_{c}} \varepsilon_{0},
$$

where $\varepsilon_{0}$ is the strain corresponding to maximum compressive stress $f_{c}$ of unconfined concrete.

The confining effect does not exist in the initial elastic stage of loading due to the fact that the Poisson ratio of concrete is lower than that of steel in this period. In this paper, it is assumed that the initial elastic modulus of confined concrete is equal to that of unconfined common concrete.

With the three parameters $f_{c c}, \varepsilon_{c c}$, and $E_{c}$, the stressstrain relation of confined concrete in the ascending branch can be described by Saenz equation [18]:

$$
\sigma=\frac{E_{0} \varepsilon}{1+\left(\frac{E_{0}}{E_{c s}}-2\right)\left(\frac{\varepsilon}{\varepsilon_{c c}}\right)+\left(\frac{\varepsilon}{\varepsilon_{c c}}\right)^{2}},
$$

where $E_{c s}$ is the secant modulus of concrete at the peak stress point, which is given as $f_{c c} / \varepsilon_{c c}$.

For the descending branch of the stress and strain relationship, an exponential degrading function is adopted [15]:

$$
\sigma=f_{c u}+\left(f_{c c}-f_{c u}\right) \exp \left(-k \frac{\varepsilon-\varepsilon_{c c}}{\varepsilon_{c c}}\right),
$$

in which $f_{c u}$ is the ultimate residual compressive stress, and $k$ is a parameter to describe the descending rate which is assumed to be $0.3 . f_{c u}$ is determined through the following equation: 


$$
f_{c u}=\left\{\begin{array}{cc}
f_{c c}\left(\frac{0.1 f_{y}}{f_{c}}\right)^{0.1} & \text { for } \frac{D}{t} \leq 40, \\
f_{c c}\left(\frac{0.1 f_{y}}{f_{c}}\right)^{0.1}\left[\lambda+(1-\lambda) \exp \left(-\frac{\frac{D}{t}-40}{40}\right)\right] & \text { for } \frac{D}{t} \geq 40,
\end{array}\right.
$$

where $\lambda$ is an empirical parameter, which is assumed to be 0.6 in this paper.

For tension of confined concrete, the tensile strength is neglected in the present study.

\subsubsection{Steel}

Under compressive load, owing to volume expansion of core concrete, steel tube will be subjected to hoop tension stress, and biaxial stress state occurs. With Von Mises criterion, the yield envelope of steel tube under biaxial stresses can be written as

$$
\sigma_{v}^{2}-\sigma_{v} \sigma_{h}+\sigma_{h}^{2}=f_{y}^{2},
$$

in which $\sigma_{v}$ is the axial stress of steel tube. With the assumption that hoop stress $\sigma_{h}$ is $0.1 f_{y}$ [19], the vertical yield stress of steel tube can be solved from Eq. (17):

$$
f_{y v}^{c} \approx 0.95 f_{y}, \quad f_{y v}^{t} \approx 1.05 f_{y},
$$

where $f_{y v}^{c}$ and $f_{y v}^{t}$ represent the axial compressive and tensile yield stress of steel tube, respectively.

\subsection{Geometrical nonlinearity}

To describe the geometrical nonlinear effect, updated Lagrangian formulation (UL) is adopted. For a large displacement motion, the virtual work equation can be written as

$$
\int_{V_{0}} S_{i j} \delta E_{i j} \mathrm{~d} V_{0}=\int_{V_{0}} p_{0 i} \delta u_{i} \mathrm{~d} V_{0}+\int_{A_{0}} q_{0 i} \delta u_{i} \mathrm{~d} A_{0},
$$

where $S_{i j}$ and $E_{i j}$ are the Kirchoff stress tensor and Green strain tensor, $p_{0 i}$ and $q_{0 i}$ is the body force and surface trace, $u_{i}$ is the displacement tensor, and $\delta$ stands for variation.

Since UL formulation is referred to the last configuration, the Green strain should be given in an incremental form, which is defined as

$$
\begin{aligned}
\Delta E_{i j} & =\Delta E_{i j}^{L}+\Delta E_{i j}^{N} \\
& =\frac{1}{2}\left(\frac{\partial \Delta u_{i}}{\partial x_{j}}+\frac{\partial \Delta u_{j}}{\partial x_{i}}\right)+\frac{1}{2} \frac{\partial \Delta u_{k}}{\partial x_{i}} \frac{\partial \Delta u_{k}}{\partial x_{j}},
\end{aligned}
$$

where $\Delta E_{i j}^{L}$ and $\Delta E_{i j}^{N}$ represent the linear and nonlinear strain, respectively. Substituting Eq. (8) into Eq. (20), the matrix form of the linear and nonlinear straindisplacement relation can be obtained

$$
\begin{aligned}
& \Delta E_{\mathrm{L}}=[\boldsymbol{B}]_{\mathrm{L}} \Delta \boldsymbol{U}, \\
& \Delta E_{\mathrm{N}}=[\boldsymbol{B}]_{\mathrm{N}} \Delta \boldsymbol{U},
\end{aligned}
$$

where $[\boldsymbol{B}]_{\mathrm{L}}$ and $[\boldsymbol{B}]_{\mathrm{N}}$ are the linear and nonlinear strain matrix, respectively. Using the virtual work equation given in Eq. (19), the geometrical stiffness matrix can be finally obtained, and the details about derivation procedure can be referred to Ref. [20].

\subsection{Arc-length method}

In the present study, the arc-length method is employed in nonlinear FEM analysis. As a displacement control algorithm, the arc-length method is more numerically stable in nonlinear finite element analysis than the Newton-Raphson approach, which may be efficient for capturing ultimate resistance capacity when soften behavior occurs.

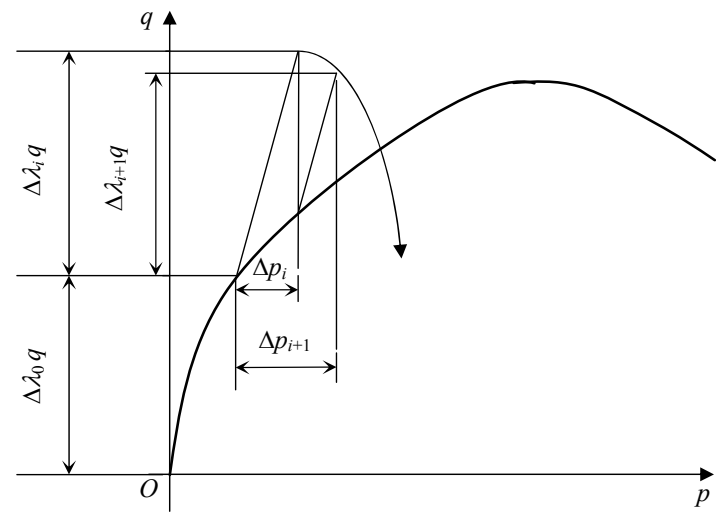

Fig. 2 Arc-length method

As shown in Fig. 2, the constraint equation of iteration can be expressed as [21]

$$
\Delta \boldsymbol{p}_{i}^{\mathrm{T}} \Delta \boldsymbol{p}_{i}+A_{0} \Delta \lambda_{i}^{2}=\Delta \boldsymbol{p}_{i+1}^{\mathrm{T}} \Delta \boldsymbol{p}_{i+1}+A_{0} \Delta \lambda^{2}{ }_{i+1}=\Delta l^{2},
$$


where $\Delta p_{i}$ is the incremental displacement after the $i$ th iteration, $\Delta \lambda_{i}$ is the incremental load factor after the $i$ th iteration, $A_{0}$ is a scalar parameter, and $\Delta l$ is the arc length.

After the $i$ th iteration, the out-of-balance force can be expressed as

$$
\boldsymbol{e}_{i}=\boldsymbol{E}_{i}-\boldsymbol{F}_{i},
$$

in which $\boldsymbol{E}_{i}$ is the external load, which can be written as $\left(\lambda_{0}+\Delta \lambda_{i}\right) \boldsymbol{q}$, and $\boldsymbol{F}_{i}$ is the internal nodal force. $e_{i}$ can be decomposed into two components

$$
g_{i}=\boldsymbol{e}_{i}^{\mathrm{T}} \boldsymbol{q} / \boldsymbol{q}^{\mathrm{T}} \boldsymbol{q}, \quad \boldsymbol{h}_{i}=\boldsymbol{e}_{i}-g_{i} \boldsymbol{q} .
$$

In the $(i+1)$ th iteration, to eliminate the out-ofbalance force, $e_{i}$ is applied on the structure. Furthermore, to satisfy Eq. (23), an extra force $\tilde{x}_{i} \boldsymbol{q}$ is also applied. Then, the incremental displacement after the $(i+1)$ th iteration is

$$
\Delta \boldsymbol{p}_{i+1}=\Delta \boldsymbol{p}_{i}+\boldsymbol{K}^{-1}\left(\boldsymbol{e}_{i}+\tilde{x}_{i} \boldsymbol{q}\right)=\Delta \boldsymbol{p}_{i}+x_{i} \boldsymbol{\delta}_{q}+\eta_{i} \boldsymbol{\delta}_{h i}
$$

where $\boldsymbol{K}$ is the tangential stiffness matrix, $\boldsymbol{\delta}_{q}=\boldsymbol{K}^{-1} \boldsymbol{q}$, $\delta_{h i}=\boldsymbol{K}^{-1} \boldsymbol{h}_{i}$, and $\eta_{i}$ is a scalar factor. Then, the incremental load factor $\Delta \lambda_{i+1}$ can be written as

$$
\Delta \lambda_{i+1}=\Delta \lambda_{i}-g_{i}+x_{i}
$$

Substituting Eqs. (26) and (27) into Eq. (23), then

$$
a x_{i}^{2}+2 b x_{i}+c=0
$$

where

$$
\begin{aligned}
& a=A_{0}+\boldsymbol{\delta}_{q}^{\mathrm{T}} \boldsymbol{\delta}_{q}, \\
& b=A_{0}\left(\Delta \lambda_{i}-g_{i}\right)+\boldsymbol{\delta}_{q}^{T}\left(\Delta \boldsymbol{p}_{i}+\eta_{i} \boldsymbol{\delta}_{h i}\right), \\
& c=A_{0}\left(\Delta \lambda_{i}-g_{i}\right)^{2}+ \\
& \left(\Delta \boldsymbol{p}_{i}+\eta_{i} \boldsymbol{\delta}_{h i}\right)^{\mathrm{T}}\left(\Delta \boldsymbol{p}_{i}+\eta_{i} \boldsymbol{\delta}_{h i}\right)-\Delta l^{2} .
\end{aligned}
$$

From Eq. (28) $x_{i}$ can be solved. Generally, two real roots can be solved from Eq. (28). A scalar product $\Delta \boldsymbol{p}_{i+1}^{\mathrm{T}} \Delta \boldsymbol{p}^{\mathrm{pr}}$ is calculated, and the root that gives the larger product is chosen ( $\Delta \boldsymbol{p}^{\mathrm{pr}}$ is the incremental displacement of the previous converged solution). Occasionally, when no real roots can be solved from Eq. (28), the pseudo line search technique is suggested by Lam and Morley [21].

With nonlinear analysis results obtained by the arclength method, two criteria are employed to determine the failure state of CFT in the present study. First, if the negative load increment is acquired by the arc-length method, the peak point of the load-displacement curve is captured, and the failure state is viewed to be achieved. On the contrary, if no softening behavior is observed, the yield of steel and crushing of concrete are chosen as criteria to judge the failure.

\section{Reliability analysis}

\subsection{The first-order reliability method (FORM)}

Approaches such as the Monte Carlo method, importance sampling method, FORM, and the second-order reliability method (SORM), are intensively used in structural reliability analysis. Generally, the failure probability can be written as

$$
P_{f}=\int_{g(x) \leq 0} f_{X}(x) \mathrm{d} x
$$

where $X$ is the vector of random variables, $f_{X}(x)$ denotes the joint probability density function of $X$, and $g(x)$ is the limit state function.

For reliability analysis by FORM, the basic random variables must be transformed into the uncorrelated standard normal space by $Y=Y(X)$. Then, Eq. (32) is rewritten as

$$
P_{f}=\int_{G(Y) \leq 0} \varphi(y) \mathrm{d} y,
$$

where $\varphi(y)$ is the standard normal density of $Y$ and $G(Y)$ is the limit state function in an uncorrelated standard normal space. Then, on the limit-state surface $G(Y)=0$, the point that has the minimum distance to the origin will be found. This point is named as the design point, which has the highest likelihood of being in the failure domain and makes the dominant contribution to the failure integral. The corresponding first-order estimate of failure probability is $P_{f}=\Phi(-\beta)$, where $\beta$, the reliability index, is the distance between the origin and design point. Therefore, the key task for FORM is to find the design point, and an effective iterative algorithm proposed in Refs. $[5,22]$ is adopted in this paper.

\subsection{Response surface method}

To find the design point, the gradient of the limit state function to random variables should be calculated. For static reliability analysis of linear or geometrical nonlinear structures, Der Kiureghian and Ke [22], and Liu and Der Kiureghian [5] derived the analytical formula of the implicit gradient of the limit state function with respect to random variables. Since the material and geometrical nonlinear finite element analysis of CFT structures will be carried, the response gradient of the limit state 
function with respect to random variables may be difficult to obtain directly, and the response surface method (RSM) is an effective way to meet this requirement.

The basic idea behind RSM is to approximate the real limit state function by an equivalent polynomial function. Compared with the traditional FORM algorithm, the search for the design point with RSM may be more effective and more tractable [23]. Moreover, such problems as numerical noise in the simulation process, nonsmooth failure surface caused by multi-criterion, and model coupling in multidisciplinary analysis, also motivate the use of the response surfaces method.

The form of the response surface is usually chosen to be of a second-order polynomial. In this paper, a second-order polynomial without cross terms is adopted:

$$
g(x)=a+\sum_{i=1}^{n} b_{i} x_{i}+\sum_{i=1}^{n} c_{i} x_{i}^{2}
$$

where $n$ is the number of random variables $X$; and $a_{0}, b_{i}$, and $c_{i i}$ are $2 n+1$ unknown coefficients to be determined. Two kinds of experimental design, saturated design, and central composite design (CCD), are usually used to determine the unknown coefficients [24-26]. In the saturated design, the required number of sampling points is the same as that of unknown coefficients, and the unknown coefficients are obtained through solving a set of linear equations. CCD requires more sampling points, and regression analysis should be introduced to determine the unknown coefficients. Compared with saturated design, CCD are more accurate but less efficient. To reduce the computational cost, the saturated design method is adopted in this paper.

\section{Example study}

\subsection{Reliability of ultimate resistance capacity of CFT column}

The reliability of resistance of a CFT column with $D \times t=216 \mathrm{~mm} \times 4 \mathrm{~mm}$ is investigated. The slenderness of the column is 18.52 . The following material properties are employed in the deterministic analysis: compressive strength of unconfined concrete $f_{c}=36.30 \mathrm{MPa}$, elastic modulus of concrete $E_{0}=32.50 \mathrm{GPa}$, strain corresponding to peak stress of unconfined concrete $\varepsilon_{0}=0.0022$, yield strength of steel $f_{y}=343.55 \mathrm{MPa}$, and elastic modulus of steel $E_{s}=20.00 \mathrm{GPa}$. The results of the resistance of column with deterministic analysis are plotted in Fig. 3.

In reliability analysis, the compressive strength of unconfined concrete $f_{c}$, yield strength of steel $f_{y}$, axial load $P$, and eccentricity $e$ are chosen as random vari- ables. $f_{c}$ and $f_{y}$ are assumed to be lognormal random variables with the following stochastic parameters:

$$
\begin{aligned}
\mu_{f_{c}} & =36.30 \mathrm{MPa}, \\
\sigma_{f_{c}} & =4.21 \mathrm{MPa}, \\
\mu_{f_{y}} & =343.35 \mathrm{MPa}, \\
\sigma_{f_{y}} & =27.47 \mathrm{MPa} .
\end{aligned}
$$

The axial load $P$ is assumed as a normal random variable with mean of $0.57 P_{u}\left(P_{u}\right.$ is the ultimate resistance capacity obtained from deterministic analysis shown in Fig. 3) and $\mathrm{COV}=0.05$. Eccentricity $e$ is assumed to be normally distributed. Fig. 4 illustrates the influences of eccentricity $e$ on the reliability index of ultimate resistance capacity.

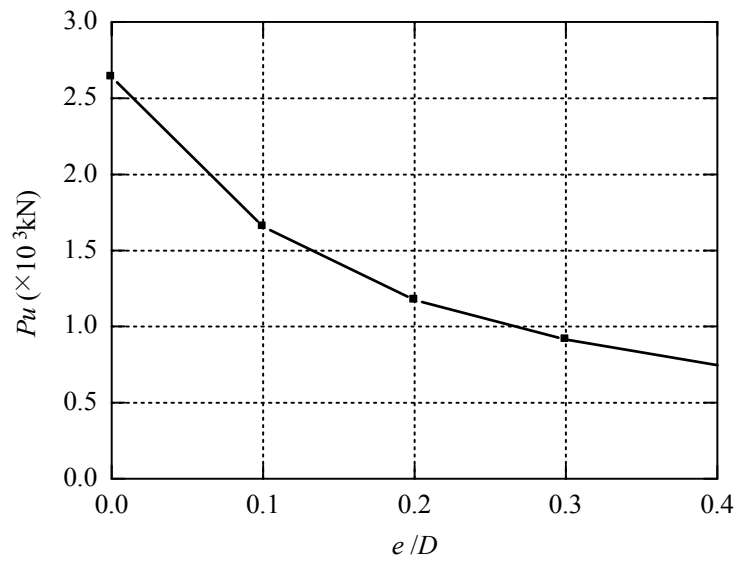

Fig. 3 Ultimate resistance capacity with deterministic analysis

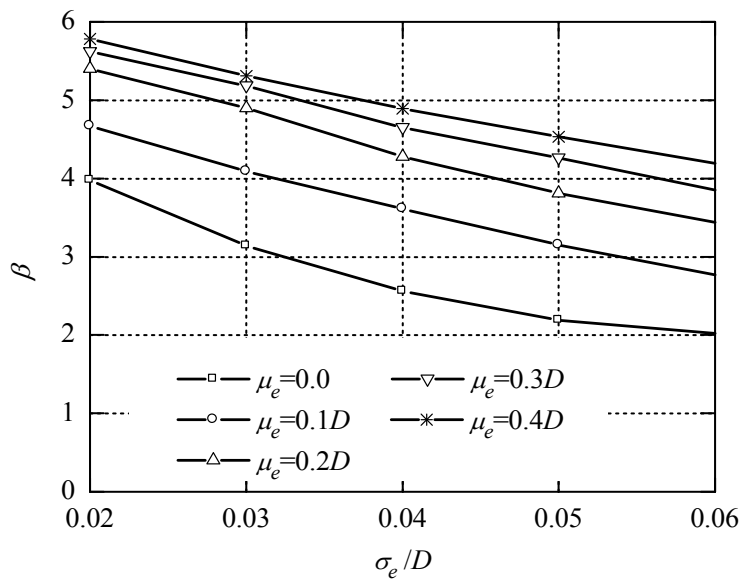

Fig. 4 Reliability of ultimate resistance capacity

It can be observed from Fig. 4 that although safety factors of ultimate resistance capacity obtained from deterministic analysis are equal, the results of the reliability index are quite different. With the increase in mean value of eccentricity, the reliability index of ultimate resistance capacity increases. Therefore, it can be deduced that it is almost impossible to define an identical safety factor for different load cases, and reliability analysis 
method should be suggested for calibration of design code of CFT structures. Fig. 5 shows the results of sensitivity $\partial \beta / \partial y$ when standard deviation of eccentricity $\sigma_{e}$ varied from $0.02 D$ to $0.06 D$. It can be viewed that with the increase in mean of eccentricity, the reliability index becomes more sensitive to the fluctuation of $f_{y}$ and

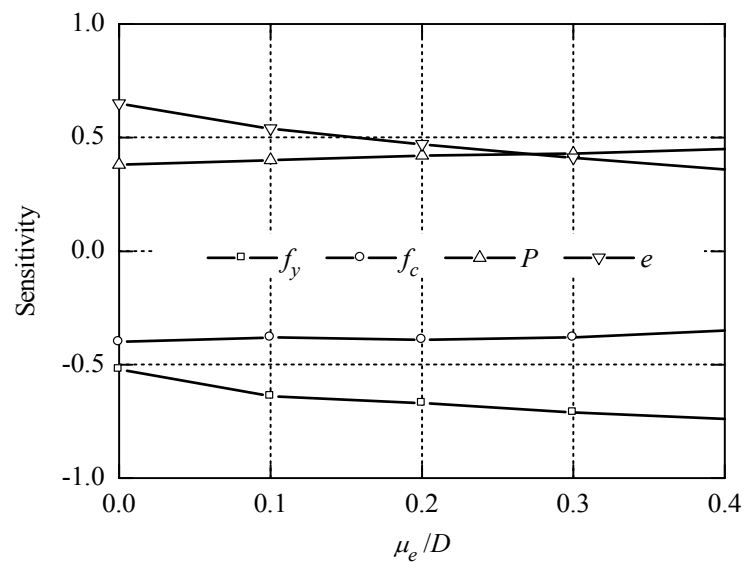

(a) $\sigma_{e}=0.02 D$

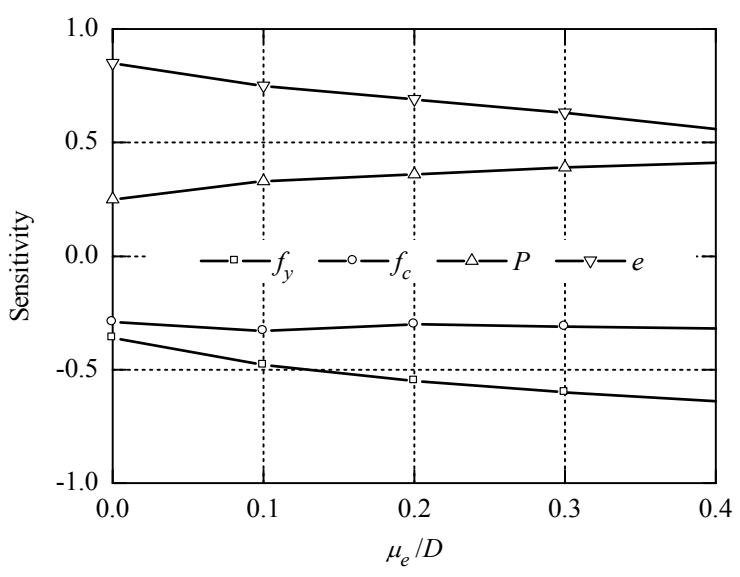

(c) $\sigma_{e}=0.04 D$ its sensitivity to $f_{c}$ remains almost unchanged. This phenomenon may be due to the fact that with the increase in eccentricity, bending moment will dominate the failure of column gradually, and as a result, the influence of uncertainty of $f_{y}$ on structure reliability enhances. More over, it is should be noted that when the mean value of $e$

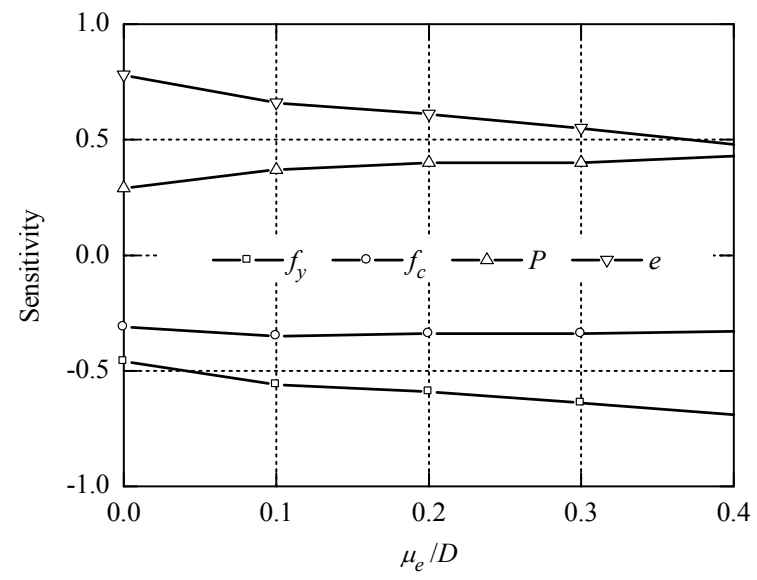

(b) $\sigma_{e}=0.03 \mathrm{D}$

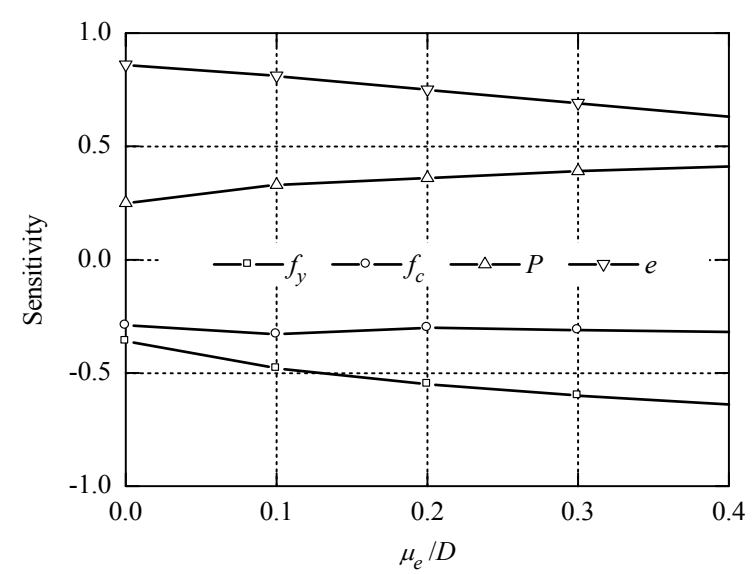

(d) $\sigma_{e}=0.05 \mathrm{D}$

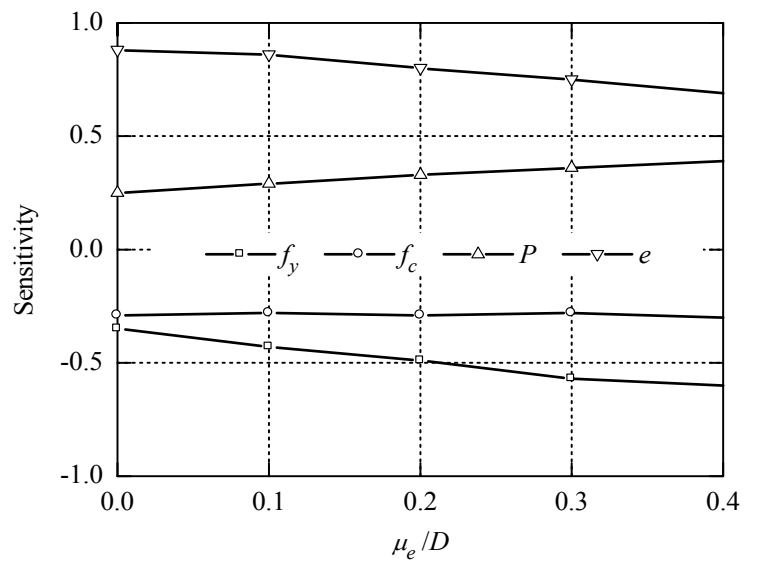

(e) $\sigma_{e}=0.06 D$

Fig. 5 Results of sensitivity 
is comparably small (i.e., concentrically loaded or small eccentrically loaded column), $e$ is overwhelmingly sensitive to the reliability index among all variables.

As shown in Fig. 5, when $\mu_{e}$ equals zero and $\sigma_{e}$ is greater than $0.02 \mathrm{D}$, uncertainty of $e$ plays a dominant role for reliability of ultimate resistance capacity, which means that randomness of eccentricity may strongly harm the structural safety in the concentrically loading case. This phenomenon should be given enough attention in analysis and design for concentrically loaded CFT column.

\subsection{Reliability of ultimate resistance capacity of imper- fect slender CFT column}

It has been widely accepted that the initial geometrical imperfection should be considered in design and analysis of slender compressive structures, especially when stability and buckling problems are concerned. To deal with the imperfection, it can be simply and conservatively assumed that the initial geometrical imperfection possess the same shape of the classical buckling mode. However, it should be worthwhile to note that the imperfection has a stochastic property in nature, and it may be rational to treat this phenomenon as a random field.

A slender concentrically loaded CFT column with simple support is studied. The geometrical configuration of the column is $L \times D \times t=5000 \mathrm{~mm} \times 216 \mathrm{~mm} \times 4 \mathrm{~mm}$. The material properties given in Example 1 are also adopted. In this example, axial load $P$ is assumed to be a deterministic variable with value of $500 \mathrm{kN}$. The imperfection is assumed as a normal random field with mean value of zero, and the standard deviation $\sigma_{I}$ is assumed to be $L / 400, L / 300$, and $L / 200$, respectively. The autocorrelation coefficient function of the random field is defined as

$$
\rho=\exp \left(-\frac{|\Delta x|}{\alpha L}\right)
$$

where $\Delta x$ is the distance between two points, $L$ is the length of the column, and $\alpha$ is a dimensionless measure of the correlation length.

Generally, for use in such reliability algorithms as FORM, the random field should be represented in terms of random variables. To meet this end, several methods have been proposed in previous studies. In this paper, the domain of the random field is discretized into a mesh of random field elements, and the random field will be described through interpolation of the random element value represented by a set of random variables. The size of the random field element is an important consideration in random field discretization. Experience has shown that a size of random field element equal to one-half to onequarter of the correlation length is appropriate $[5,22]$. In the present example, the column is discretized into 9 random field elements with equal length.

Fig. 6 shows the reliability results of ultimate resistance when $\alpha$ varies from 0.1 to 0.6 . From Fig. 6, it can be found that with the increase in standard deviation of initial geometrical imperfection, the reliability of slender CFT column decreases enormously. Moreover, it can also be viewed that with the increase in $\alpha$, i. e., the random field approaches the random variable gradually, the reliability index decreases gradually.

Fig. 7 presents the geometrical imperfection at the design point when $\alpha=0.01$. It can be observed that the geometrical imperfection found at the design point is similar to the density function subject to the normal distribution, which is distinctively different from the imperfection with sine curve assumed in deterministic analysis.

To give a further discussion about this phenomenon, Fig. 8 gives the comparison of ultimate resistance capacity when imperfection is sine, triangular, and the present

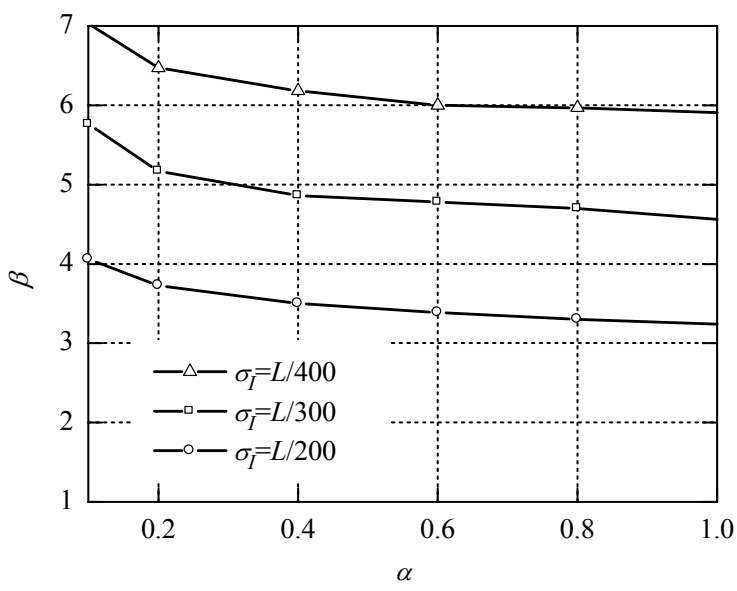

Fig. 6 Influence of imperfection on reliability index

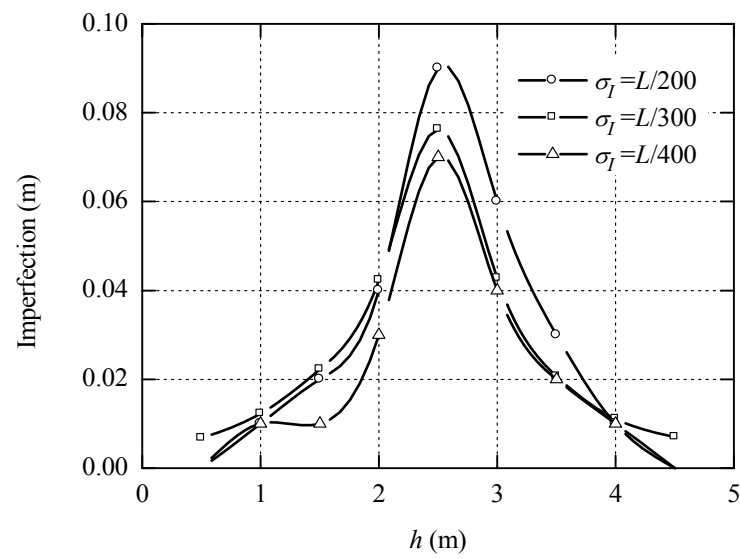

Fig. 7 Geometrical imperfection at design point 


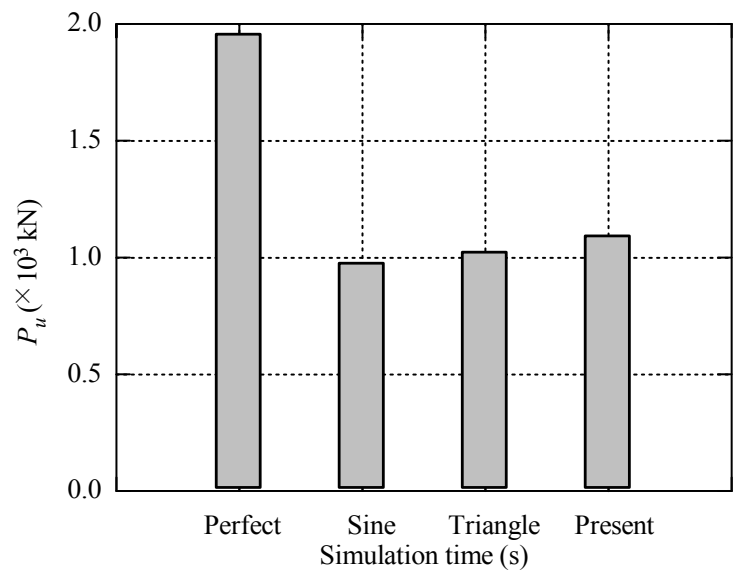

Fig. 8 Ultimate resistance capacity

form with the same peak value of $L / 200$.

Although ultimate resistance capacity obtained from the present imperfection is about 1.12 times that computed with sine imperfection mode, the present imperfection has a higher likelihood of being in the failure domain, which should be paid more attention to imperfection control during construction process.

\section{Conclusions}

In this paper, a systematical approach for reliability analysis of CFT structures through combining FORM and nonlinear FEM is presented. Geometrical and material nonlinearity are involved in nonlinear analysis, and a modified constitutive model for core concrete is adopted to simulate the confining effect of CFT structures. Through combining FORM and RSM, the reliability of ultimate resistance capacity of CFT columns is evaluated.

Through a numerical example study, the influence of such parameters as material strength, eccentricity, slenderness, geometrical imperfection, etc., on the reliability of CFT columns is investigated. It can be concluded that inevitable random fluctuation of those parameters has significant influences on structural reliability, and that stochastic or reliability methods can give a more rational and subjective evaluation on the safety of CFT structures than a deterministic approach. Although the research of this paper focuses mainly on evaluating the CFT columns, the reliability of other CFT structures can also be obtained by following the same procedure.

\section{Acknowledgements}

This paper was financially supported by the Fundamental Research Funds for the Central Universities (SWJTU09CX012 and SWJTU11BR006), and the Doc- toral Fund for Youth Scholars of Ministry of Education of China (No. 20110184120010).

\section{References}

[1] L.H. Han, Concrete-Filled Steel Tubular Structures, Beijing: Science Publishing House, 2000 (in Chinese).

[2] O. Ditlevsen, H.O. Madsen, Structural Reliability Method, New York: John Wiley \& Sons, 1996.

[3] R.E. Melchers, Structural Reliability Analysis and Prediction, New York: John Wiley \& Sons, Inc, 1999.

[4] H. Haldar, S. Mahadevan, Reliability Assessment Using Stochastic Finite Element Analysis, New York: John Wiley \& Sons, 2000.

[5] P.L. Liu, A.D. Kiureghian, Finite element reliability of geometrical nonlinear uncertain structures, Journal of Engineering Mechanics, 1991, 117(8): 1806-1825.

[6] K. Imai, D.M. Frangopol, Geometrically nonlinear finite element reliability analysis of structural systems, I: theory, Computers and Structures, 2000, 77(6): 677691.

[7] N. Impollonia, A. Sofi, A response surface approach for the static analysis of stochastic structures with geometrical nonlinearities, Computer Methods in Applied Mechanics and Engineering, 2003, 192(37-38): 4109-4129.

[8] D.M. Frangopol, Y. Ide, E. Spacone, et al., A new look at reliability of reinforced concrete columns, Structural Safety, 1996, 18(2-3): 123-150.

[9] J.G. Teigen, D.M. Frangopol, S. Sture, et al., Probabilistic FEM for nonlinear concrete structures. I: theory, Journal of Structural Engineering, 1991, 117(9): 26742689.

[10] F. Biondini, F. Bontempi, D.M. Frangopol, et al., Reliability of material and geometrically non-linear reinforced and prestressed concrete structures, Computers and Structures, 2004, 82(13-14): 1021-1031.

[11] T.H. Lee, K.M. Mosalam, Probabilistic fiber element modeling of reinforced concrete structures, Computers and Structures, 2004, 82(27): 2285-2299.

[12] D. Val, F. Blgujer, D. Yankelevsky, Reliability evaluation in nonlinear analysis of reinforced concrete structures, Structural Safety, 1997, 19(2): 203-217.

[13] R.C. Soares, A. Mohamed, W.S. Venturini, et al., Reliability analysis of non-linear reinforced concrete frames using the response surface method, Reliability Engineering and System Safety, 2002, 75(1): 1-16.

[14] T.Y. Xiang, Y.Q. Tong, R.D. Zhao, A general and versatile nonlinear analysis program for concrete bridge structure, Advances in Engineering Software, 2005, 36(10): 681-690.

[15] T.F. Xu, T.Y. Xiang, R.D. Zhao, et al., Nonlinear finite element analysis of circular concrete-filled steel tube structures, Structural Engineering and Mechanics, 2010, 35(3): 315-334.

[16] H.T. Hu, C.S. Huang, M.H. Wu, et al., Nonlinear analysis of axially loaded concrete-filled tube columns with confinement effect, Journal of Structural Engineering, 
2003, 129(10): 1322-1329.

[17] B.C. Chen, Z.P. Ou, L.Y. Wang, et al., Experimental study on carrying capacity of concrete filled steel tubular column under eccentric load, Journal of Fuzhou University, 2002, 30(6): 838-844 (in Chinese).

[18] P. Desayi, S. Krishnan, Equation for the stress-strain curve of concrete, Journal of ACI, 1964, 61(3): 12291235.

[19] A. Elremaily, A. Azizinamini, Behavior and strength of circular concrete-filled tube columns, Journal of Constructional Steel Research, 2002, 58(12): 1567-1591.

[20] K.J. Bathe, S. Bolourchi, A geometrical and material nonlinear plate and shell element, Computers and Structures, 1980, 11: 23-48.

[21] W.F. Lam, C.T. Morley, Arc-length method for passing limit points in structural calculation, Journal of Structural Engineering, 1992, 118(1): 169-185.
[22] A.D. Kiureghian, J.B. Ke, The stochastic finite element method in structural reliability, Probabilistic Engineering Mechanics, 1988, 3(2): 83-91.

[23] X.S. Nguyen, A. Sellier, F. Duprat, et al., Adaptive response surface method based on a double weighted regression technique, Probabilistic Engineering Mechanics, 2009, 24(2): 135-143.

[24] C.G. Bucher, U. Bourgund, A fast and efficient response surface approach for structural reliability problems, Structural Safety, 1990, 7(1): 57-66.

[25] J. Huh, A. Haldar, Stochastic finite-element-based seismic risk of nonlinear structures, Journal of Structural Engineering, 2001, 127(3): 323-329.

[26] M.R. Rajashkhar, B.R. Ellingwood, A new look at the response surface approach for structural reliability analysis, Structural Safety, 1993, 12(3): 205-220.

(Editor: Dongju CHEN) 